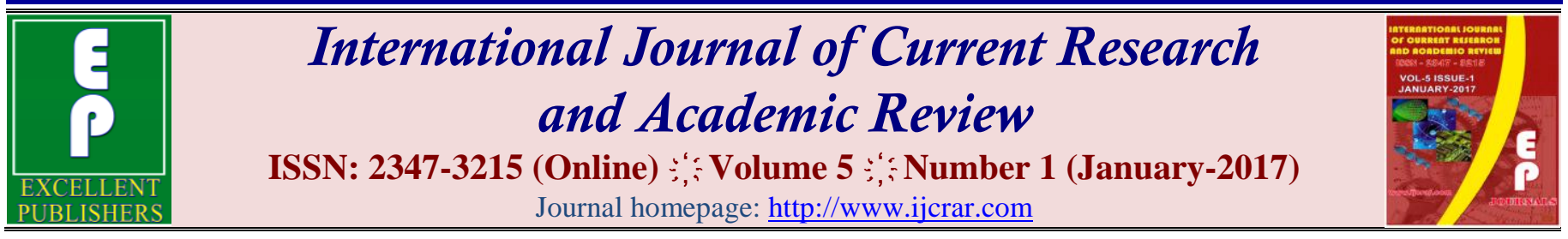

doi: http://dx.doi.org/10.20546/ijcrar.2017.501.010

\title{
Analysis, Modelling and Simulation of Co-operative OFDM System to implement Amplify and Forward transmission in wireless Relay Communications
}

\author{
Anuja Shirke and S.V. Patil* \\ EXTC Department, J.T. Mahajan College of Engineering, Faizpur, India \\ *Corresponding author.
}

\begin{abstract}
Orthogonal Frequency Division Multiplexing (OFDM) is one of the latest modulation techniques used in order to combat the frequency-selectivity of the transmission channels, achieving high data rate without inter-symbol interference. The basic principle of OFDM is gaining a wide spread popularity within the wireless transmission community. Furthermore, OFDM is one of the main techniques proposed to be employed in 4th Generation Wireless Systems. Therefore, it is crucial to understand the concepts behind OFDM. In this paper it is given an overview of the basic principles on which this modulation scheme is based with fundamental results using 4-QAM \& QPSK using AWGN, Rayleigh and rician channels.
\end{abstract}

\section{Article Info}

Accepted: 10 January 2017

Available Online: 20 January 2017

\section{Keywords}

OFDM, 4-QAM, QPSK, AWGN channel, Raleigh channel, Rician channel, DFT, Cooperative wireless Communication.

\section{Introduction}

\section{Cooperative relay communications}

Signal fading due to multi-path propagation is one of the major impairments to meet the demands of next generation wireless networks for high data rate services. To mitigate the fading effects, time, frequency, and spatial diversity techniques or their hybrid can be used. Among different types of diversity techniques, spatial diversity is of special interest as is does not incur system losses in terms of delay and bandwidth efficiency. Recently, cooperative diversity in wireless network has received great interest and is regarded as a promising technique to mitigate multi-path fading, which results in a fluctuation in the amplitude of the received signal. The cooperative communications is a new communication paradigm which generates independent paths between the user and the base station by introducing a relay channel. The relay channel can be thought of as an auxiliary channel to the direct channel between the source and destination. The basic idea behind cooperation is that several users in a network pool their resources in order to form a virtual antenna array which creates spatial diversity (Laneman et al., 2004; Sendonaris et al., Part I, 2003; Sendonaris et al., Part II, 2003). Since the relay node is usually several wavelengths distant from the source, the relay channel is guaranteed to fade independently from the direct channel, which introduces a full-rank Multiple-inputmultiple-output (MIMO) channel between the source and the destination. This cooperative spatial diversity leads to an increased exponential decay rate in the error probability with increasing signal-to-noise ratio (SNR) (Liu et al., 2009). 


\section{Introduction to OFDM}

Due to the spectacular growth of the wireless services and demands during the last years, the need of a modulation technique that could transmit high data rates at high bandwidth efficiency strongly imposed. The problem of the inter-symbol interference (ISI) introduced by the frequency selectivity of the channel became even more imperative once the desired transmission rates dramatically grew up. Using adaptive equalization techniques at the receiver in order to combat the ISI effects could be the solution, but there are practical difficulties in operating this equalization in realtime conditions at several $\mathrm{Mb} / \mathrm{s}$ with compact, low-cost hardware. OFDM is a promising candidate that eliminates the need of very complex equalization. In a conventional serial data system, the symbols are transmitted sequentially, one by one, with the frequency spectrum of each data symbol allowed to occupy the entire available bandwidth. A high rate data transmission supposes very short symbol duration, conducing at a large spectrum of the modulation symbol. There are good chances that the frequency selective channel response affects in a very distinctive manner the different spectral components of the data symbol, hence introducing the ISI. The same phenomenon, regarded in the time domain consists in smearing and spreading of information symbols such, the energy from one symbol interfering with the energy of the next ones, in such a way that the received signal has a high probability of being incorrectly interpreted. Intuitively, one can assume that the frequency selectivity of the channel can be mitigated if, instead of transmitting a single high rate data stream, we transmit the data simultaneously, on several narrowband sub channels (with a different carrier corresponding to each Sub-channel), on which the frequency response of the channel looks "flat" (see in figure). Hence, for a given overall data rate, increasing the number of carriers reduces the data rate that each individual carrier must convey, therefore lengthening the symbol duration on each subcarrier. Slow data rate (and long symbol duration) on each sub-channel merely means that the effects of ISI are severely reduced. This is in fact the basic idea that lies behind OFDM. Transmitting the data among a large number of closely spaced subcarriers accounts for the "frequency division multiplexing" part of the name. Unlike the classical frequency division multiplexing technique, OFDM will provide much higher bandwidth efficiency. This is due to the fact that in OFDM the spectra of individual subcarriers are allowed to overlap. In fact, the carriers are carefully chosen to be orthogonal one another. As it is well known, the orthogonal signals do not interfere, and they can be separated at the receiver by correlation techniques. The orthogonality of the subcarriers accounts for the first part of the OFDM name.

\section{OFDM Basics}

The basic principle of OFDM is to divide the high-rate data stream into many low rate streams that each is transmitted simultaneously over its own subcarrier orthogonal to all the others. Due to narrowband property, they experience mostly flat fading, which makes channel equalization very simple. In order to eliminate intersymbol interference (ISI) and inter-carrier interference (ICI) as much as possible, it is a good idea to add a trailing portion of each symbol to the head of itself, which is called cyclic prefix extension as shown in Figure. The guard interval is chosen larger than the expected delay spread, such that multipath components from one symbol will not interfere with its succeeding symbol. After a signal passes through the time-dispersive channel, orthogonality of its subcarrier components can be maintained by the introduction of cyclic prefix.

\section{The concept of multicarrier (parallel) transmission}

In a mobile radio environment, the signal is carried by a large number of paths with different strength and delays. Such multipath dispersion of the signal is commonly referred as „channel-induced ISI” and yields the same kind of ISI distortion caused by an electronic filter In fact, the multipath dispersion leads to an upper limitation of the transmission rate in order to avoid the frequency selectivity of the channel or the need of a complex adaptive equalization in the receiver. In order to mitigate the time-dispersive nature of the channel, the finding of the multicarrier technique was to replace a single-carrier serial transmission at a high data rate with a number of slower parallel data streams. Each parallel stream will be then used to sequentially modulate a different carrier. By creating $\mathrm{N}$ parallel sub-streams, we will be able to decrease the bandwidth of the modulation symbol by the factor of $\mathrm{N}$, or, in other words, the duration of a modulation symbol is increased by the same factor. The summation of all of the individual sub-channel data rates will result in total desired symbol rate, with the drastic reduction of the ISI distortion. The price to pay is of course very important, since the multicarrier transmission seems to act as a frequency multiplexation, which will generate problems in terms of bandwidth efficiency usage. The things go however better than seemed, because in OFDM the carriers are orthogonal to 
each-other and they are separated by a frequency interval of $\Delta \mathrm{f}=1 / \mathrm{T}$. The frequency spectrum of the adjacent subchannels will overlap one another, but the carrier's orthogonality will eliminate in principle the inter-channel interference that we feared of.

The input data sequence is baseband modulated, using a digital modulation scheme. Various modulation schemes could be employed such as BPSK, QPSK (also with their differential form) and QAM with several different signal constellations. There are also forms of OFDM where a distinct modulation on each sub-channel is performed (e.g. transmitting more bits using an adequate modulation method on the carriers that are more „confident”, like in ADSL systems). Also, data can be encoded ,in frame" (the baseband signal modulation is performed on the serial data, that is inside of what we name a „DFT frame”), or ,inter frame” (the modulation is performed on each parallel sub-stream, that is on the symbols belonging to adjacent DFT frames). The data symbols are parallelized in $\mathrm{N}$ different sub-streams. Each sub-stream will modulate a separate carrier through the IFFT modulation block, which is in fact the key element of an OFDM scheme, as we will see later. A cyclic prefix is inserted in order to eliminate the inter-symbol and inter-block interference (IBI). This cyclic prefix of length $\mathrm{L}$ is a circular extension of the IFFT-modulated symbol, obtained by copying the last L samples of the symbol in front of it. The data are back-serial converted, forming an OFDM symbol that will modulate a highfrequency carrier before its transmission through the channel. The radio channel is generally referred as a linear time-variant system. To the receiver, the inverse operations are performed: the data are down-converted to the baseband and the cyclic prefix is removed. The coherent FFT demodulator will ideally retrieve the exact form of transmitted symbols. The data are serial converted and the appropriated demodulation scheme will be used to estimate the transmitted symbols.

\section{Modelling Principle}

The baseband discrete-time representation of OFDM. For a more general presentation based on orthogonal transmultiplexers or block pre-coding issues. The history of multi-carrier modulation began more then 30 years ago. At the beginning, only analog design based on the use of orthogonal waveforms was proposed. The use of discrete
Fourier Transform (DFT) for modulation and demodulation was first proposed in. Only recently has it been finding its way into commercial use, as the recent developments in technology have lowered the cost of the signal processing that is needed to implement OFDM systems we first give a brief overview of frequency selective channels

Let $r(t)$ be the low-pass received signal:

$$
r(t)=\int_{-\infty}^{\infty} c(\tau) x(t-\tau) d \tau+n(t) .
$$

Frequency selectivity occurs whenever the transmitted signal $x(t)$ occupies an interval bandwidth $[; W 2 ; W 2]$ greater then the coherence bandwidth Bcoh of the channel $c(t)$ (defined as the inverse of the delay spread $T d$ [8]). In this case, the frequency components of $x(t)$ with frequency separation exceeding Bcoh are subject to different gains. Results presents a typical time impulse response $c(t)$ of a channel. For usual high data rates schemes, the symbol rate $T$ is small compared to $T d$ (they are also called broadband signals) and the signals are therefore subject to frequency selectivity. The multipathchannel can be modeled by an impulse response given by

$c(t)=\sum_{l=0}^{M-1} \lambda_{l} g\left(t-\tau_{l}\right)$

The multipath-channel can be modeled by an impulse response given by $c(t)=\mathrm{P} M ; 1 l=0, l g\left(t_{i} ; l\right)$ where $g(t)$ is transmitting filter and $T d$ is the duration of the multipath or delay spread. Here, the complex gains $(, l) l=0 \ldots \ldots \ldots M_{i} 1$ are the multi-path gains and the $(i l) l=0 \ldots \ldots .(M ; 1)$ are the corresponding time delays. The variance of each gain as well as the time delays are usually determined form propagation measurements. As a typical example, the delay profile of indoor channels $\mathrm{A}$ and E. Let $W$ denote the signal bandwidth and $T=1 / W$ the sampling rate. We will assume hereafter that the transmitting filter is supposed to be ideal $(G(f)=1$ for and 0 outside, $G(f)$ being the Fourier transform of the transmitting filter $g(t)$ ).

$f \in\left[-\frac{W}{2}, \frac{W}{2}\right]$ 


\section{Simulation Results}

\section{4-QAM over AWGN Channel with Co-operative OFDM}

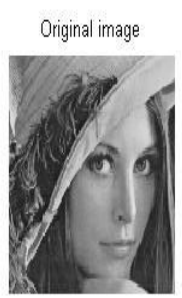

Received image direct link with 4-OAM

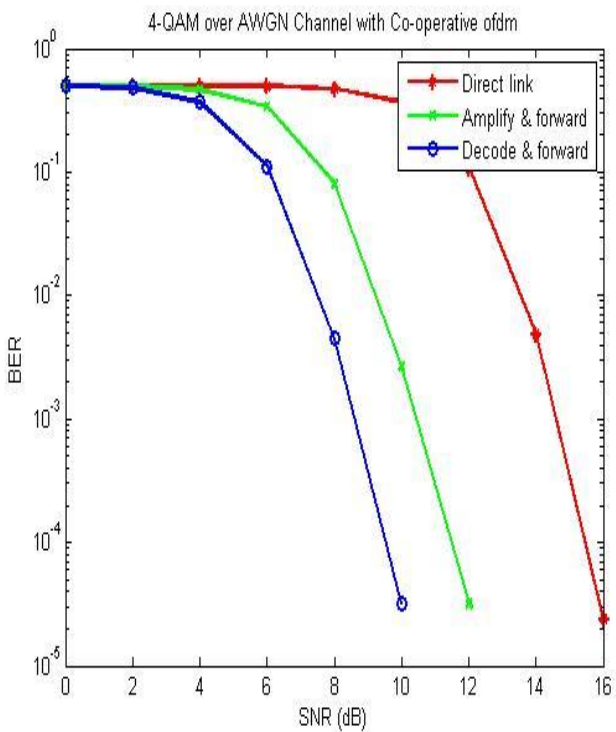

\section{4-QAM over Rayleigh Channel with Co-operative OFDM}
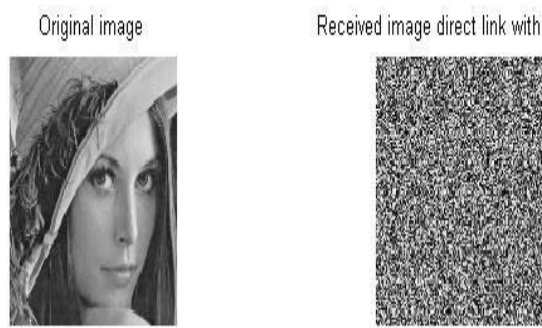

Received image Amplify and forward with 4-QAM Received image Decode and forward with 4-QAM
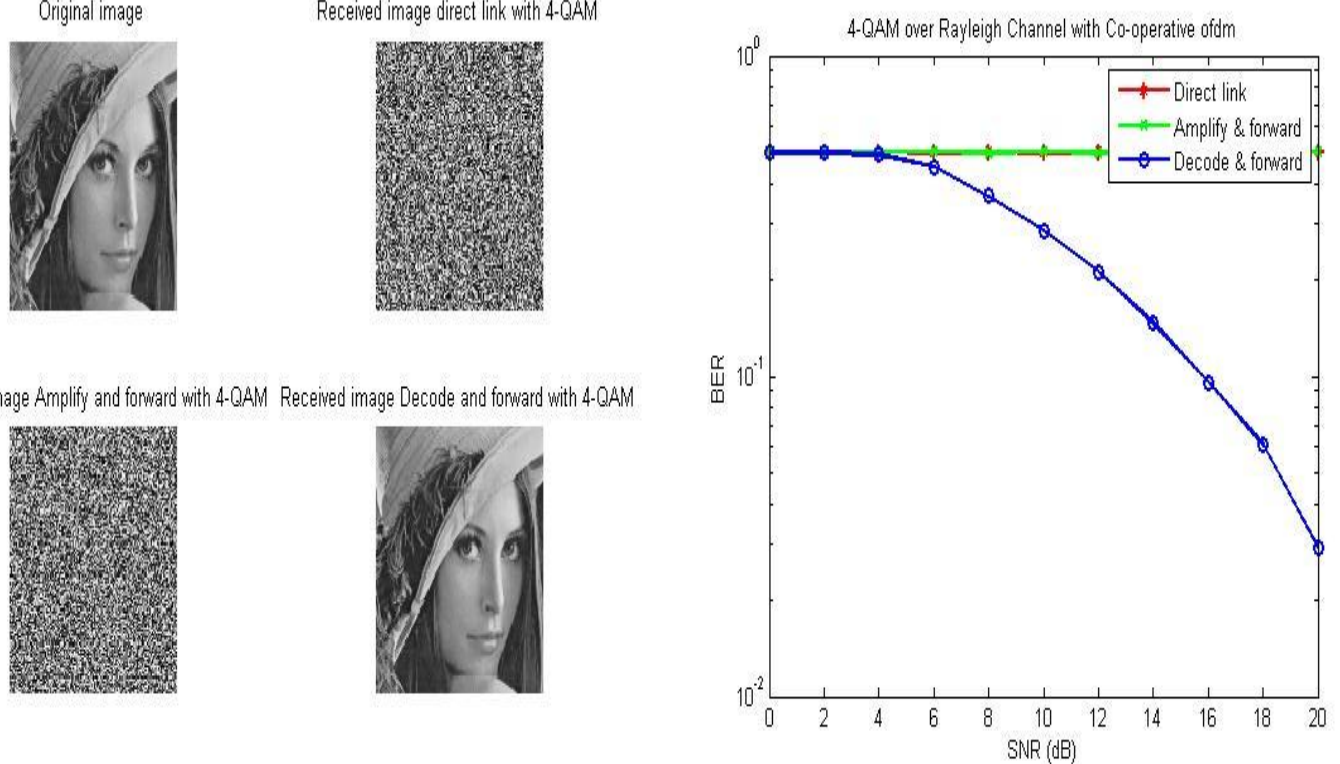


\section{4-QAM over Rician Channel with Co-operative OFDM}

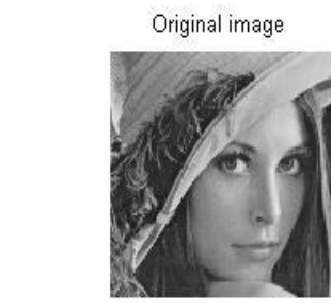

Received image direct link with 4-QAM

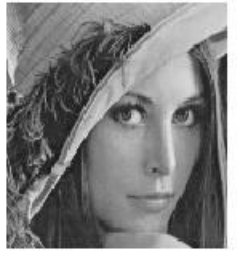

Received image Amplify and forward 4-QANReceived image Decode and forward 4-QAM
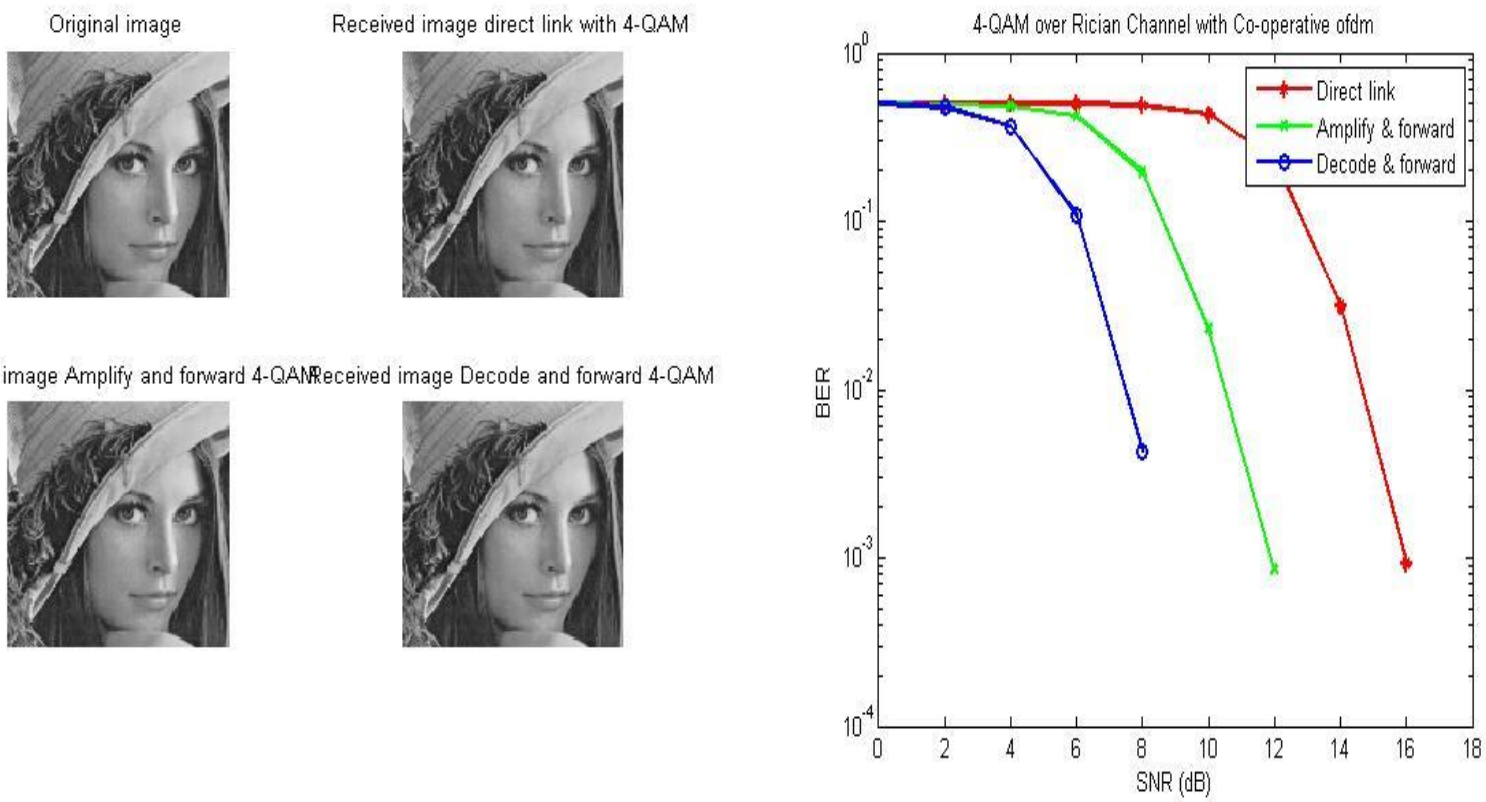

\section{QPSK over AWGN Channel with Co-operative OFDM}

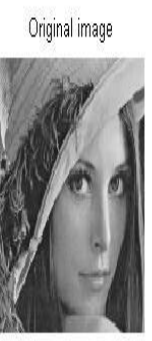

Receved image Amplify and fowward with QPSK

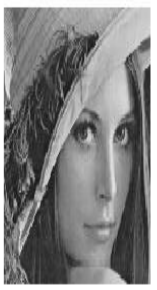

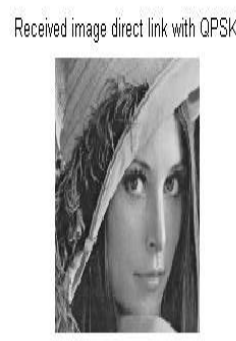

Received image Decode and foward with OPSK

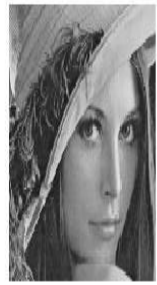

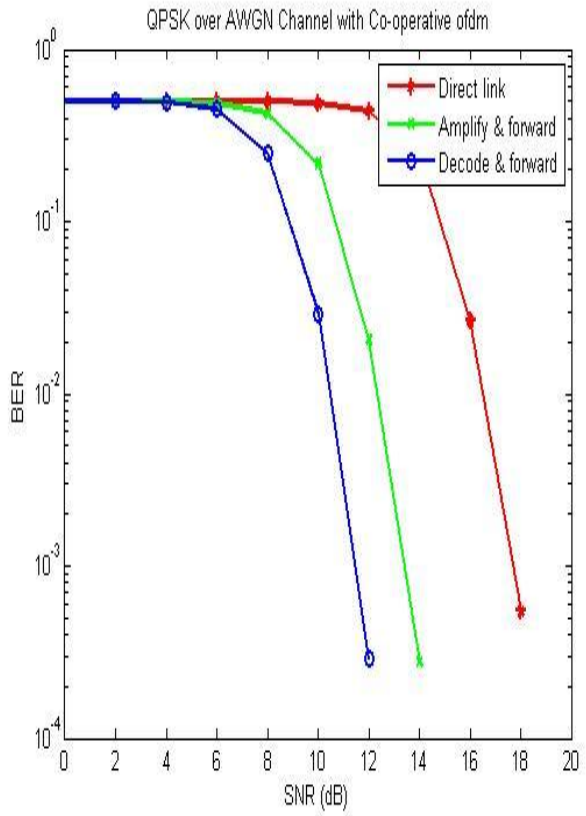




\section{QPSK over Rayleigh Channel with Co-operative OFDM}
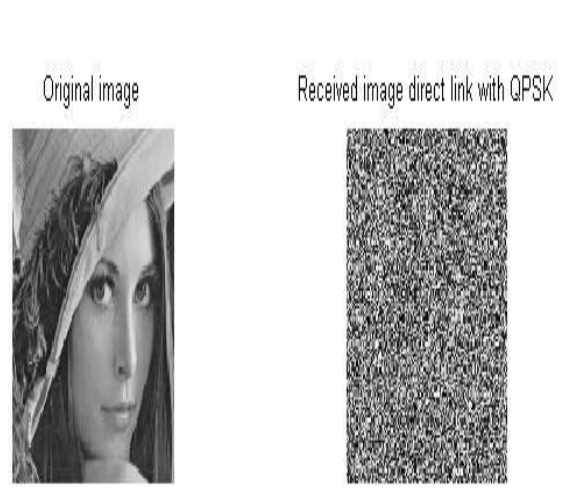

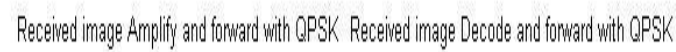
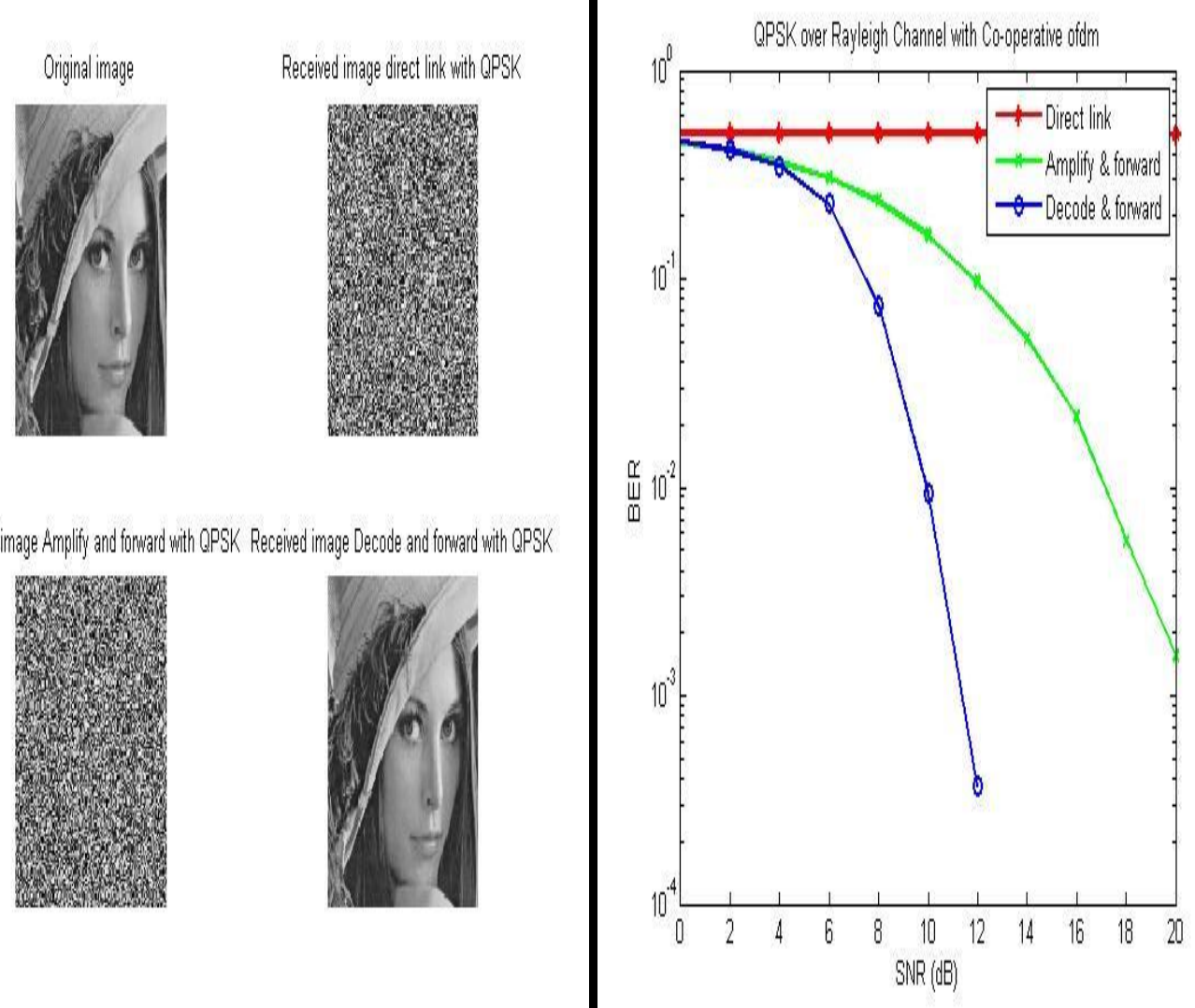

\section{QPSK over Rician Channel with Co-operative OFDM}

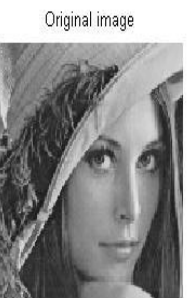

Received image Amplify and forward with QPSK

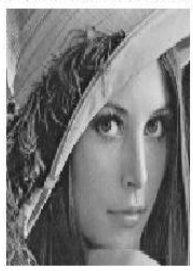

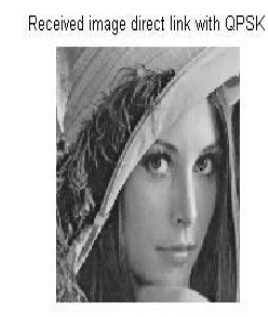

Received image Decode and forward with QPSK

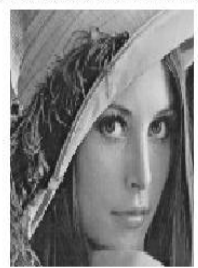

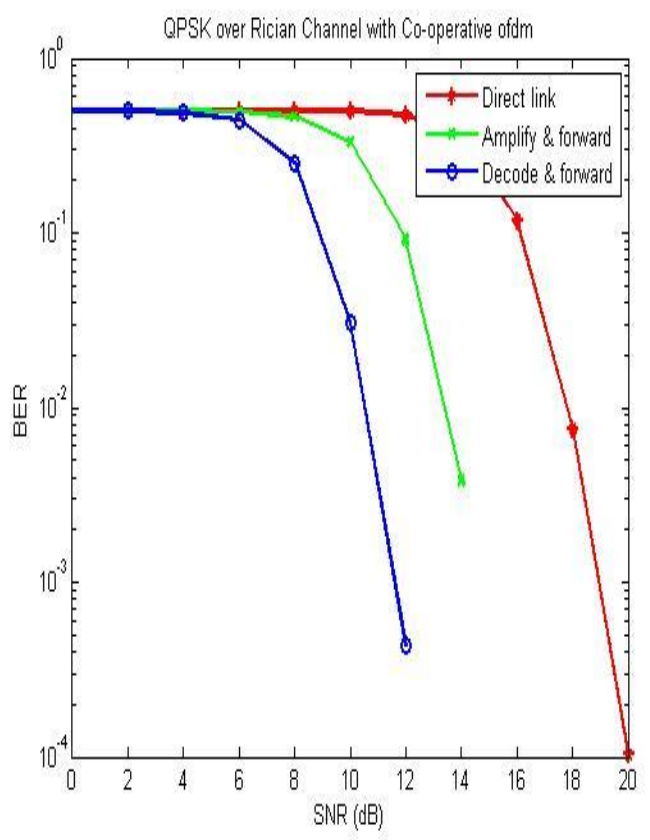

results for three different conditions direct link amplify $\&$ forward and decode \& forward. We have verified all these results for co-operative OFDM and all the simulations exactly matched with standard results.
Above simulation results for different set of parameters and frames using 4-QAM and QPSK for Bit Error Rate (BER) per frame transmission over AWGN, Rayleigh and Rician Channels for SNR in dB. We have verified 


\section{OFDM Challenges}

A pertinent conclusion regarding OFDM performances and capabilities cannot be given without a brief review of the main drawbacks and challenges that this technique must deal with. There are, for example, major practical difficulties to achieve real time synchronization for OFDM frames.

Fig 1. The frequency-selective channel response and the relatively flat response on each sub-channel
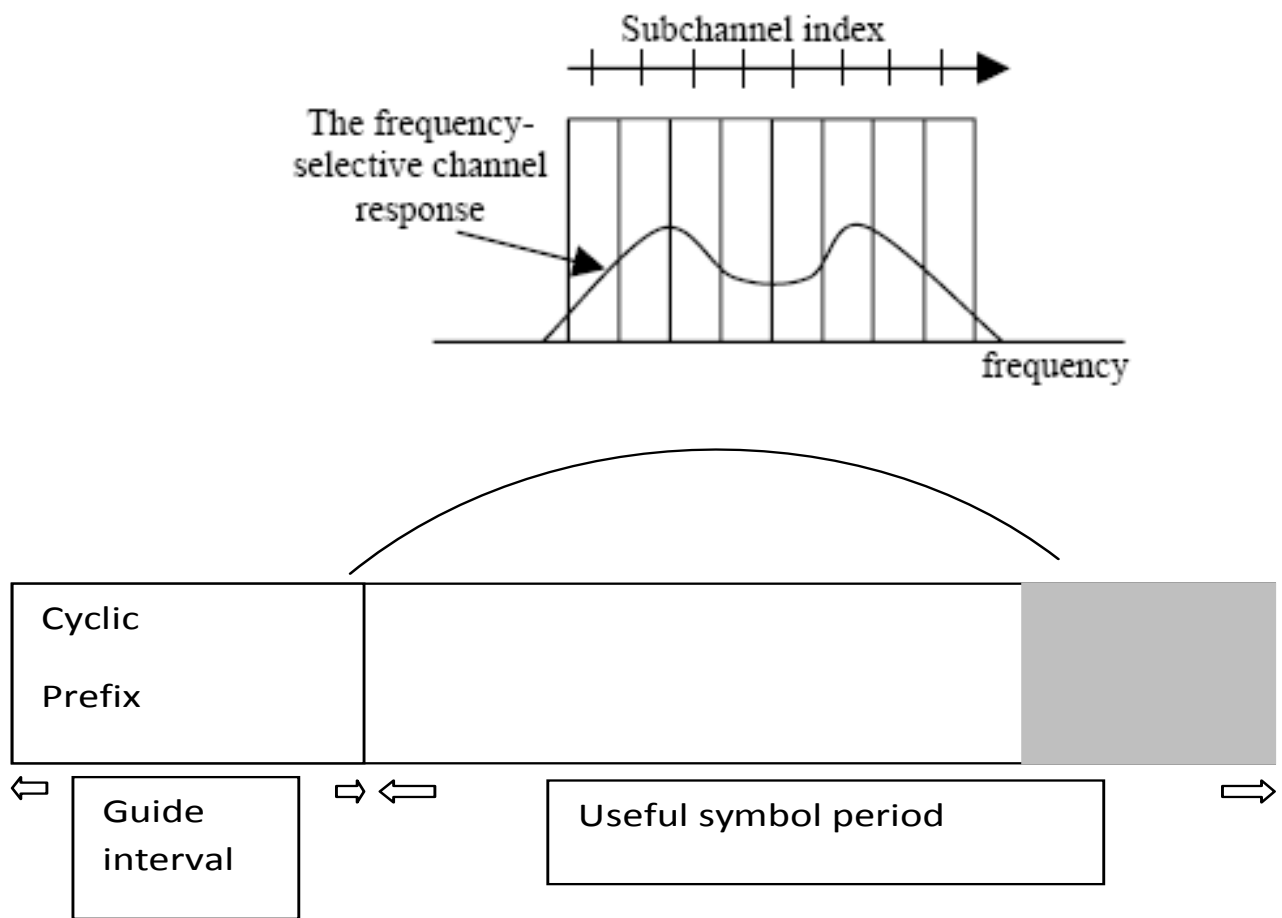

Fig: cyclic prefix extension

Wide-band channel
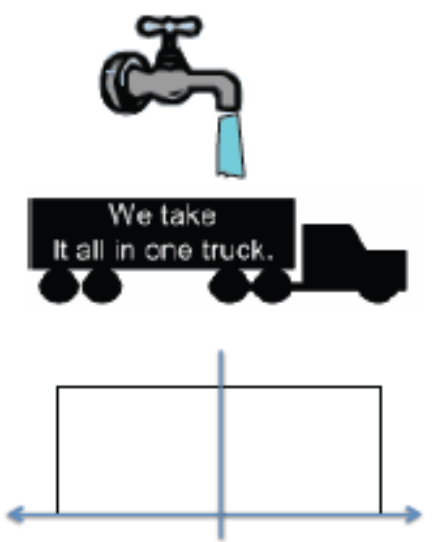

Send a sample using the entire band
Multiple narrow-band channels
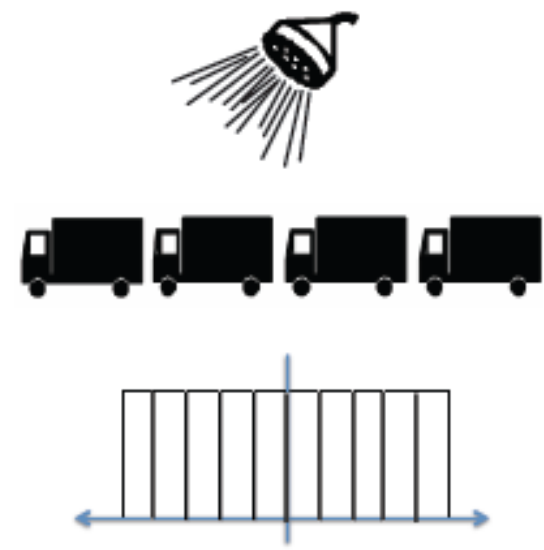

Send samples concurrently using multiple orthogonal sub-channels

Fig: OFDM Concept 


\section{OFDM functional Diagram}
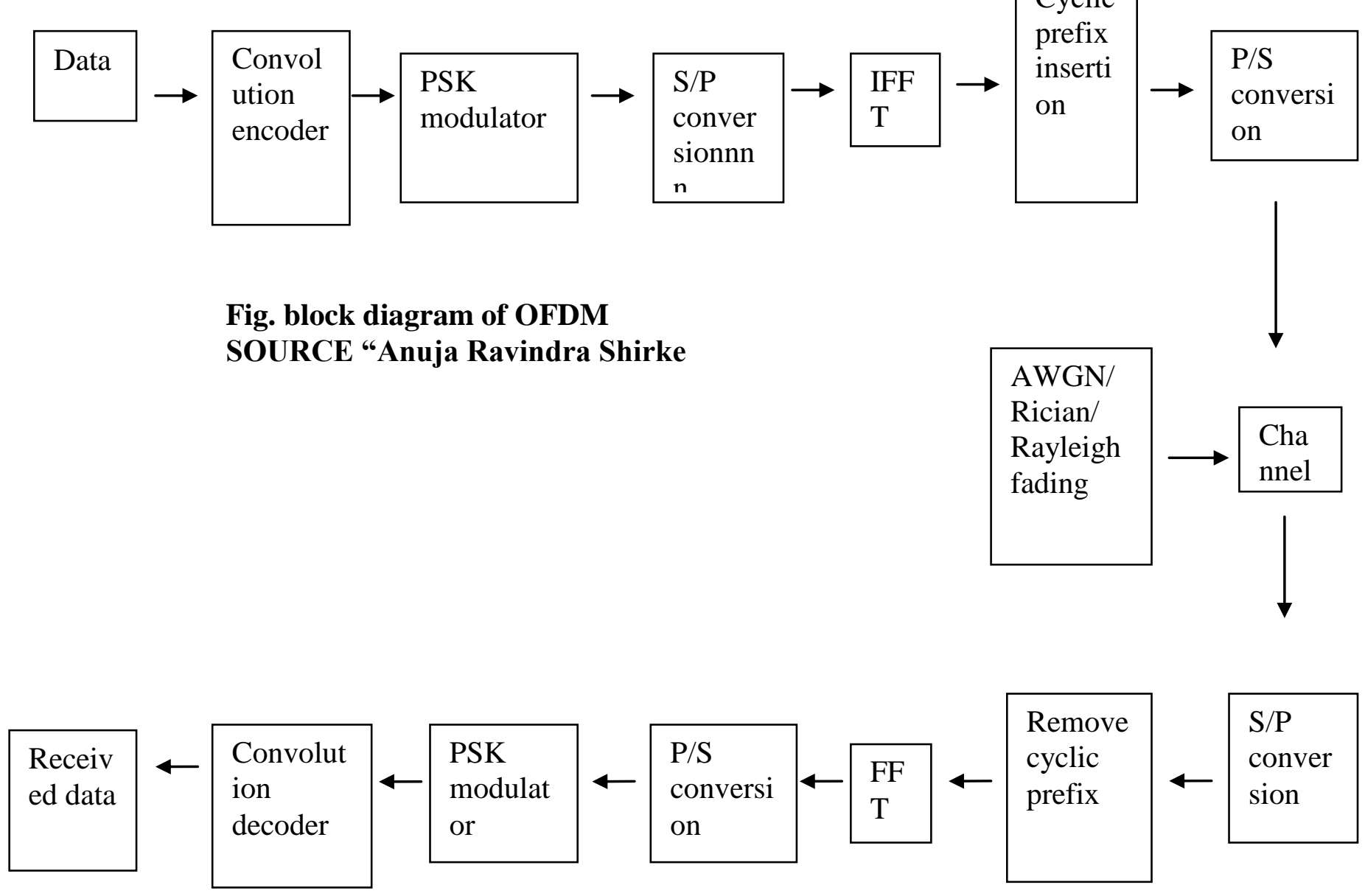

On the other hand, the technique is extremely sensitive to the frequency offsets that could cause inter-carrier interference. Also, spectral nulls in the useful transmission band will conduce to severe performance degradation on the affected sub-carriers. OFDM symbols have a high peak-to-average power ratio (PAPR) that makes them unsuitable for RF amplifiers, which could "clip" the signal peaks, hence causing distortion. Finally, but not last, full capabilities of OFDM can be achieved only if the channel impulse response is known, assumption that is not always met; complex channel estimation techniques must be used in order to achieve this need.

\section{Conclusion}

OFDM is a grouping of multiplexing as well as modulation. We studied that in an OFDM system, a large number of orthogonal, overlapping, narrow band subcarriers are transmitted in parallel. These carriers separate the obtainable transmission bandwidth. We review the OFDM system and Cooperative techniques which used in the wireless communication. In the OFDM system we overviewed the different techniques used in OFDM systems which are used in wireless communication for better communication. The simulation results shows the bit error rate performance comparison between direct transmission, OFDM transmission, Amplify and forward relaying, AFOFDM system and coded OFDM system. As can be seen from the result coded OFDM system gives a better performance over the amplify and forward-OFDM system as the noise is not getting amplified and the redundancy term added checks and corrects the error before retransmitting the re-encoded message at the relay node. However the performance of AF and AF-OFDM system are almost the same as the noise also gets amplified. This paper is a study on applying Cooperative Diversity to OFDM system. The result of simulation demonstrates that the OFDM system has a better performance when the cooperation node employs coded cooperative scheme. In the simulation, Rayleigh, Rician and AWGN multi-paths fading channel, convolution coding maximum ratio combining has been considered. 
The simulation results show that the OFDM system with coded Cooperative relaying has a better performance over the AF cooperative scheme. Comparative studies between 4-QAM \& QPSK open new opportunities for co-operative wireless relay communication.

\section{References}

Bingham, J.A.C. 1990. Multicarrier modulation for data transmission: an idea whose time has come, IEEE Commun. Mag., pp. 5-14.

Chethan, B., Ravisimha, B.N., M.Z. Kurian. The effects of Inter Symbol Interference (ISI) and FIR Pulse Shaping Filters: A survey", Int. J. Adv. Res. Electrical, Electronics and Instrumentation Engi., (An ISO 3297: 2007 Certified Organization) Vol. 3, Issue 5, pp.9411-9416.

Dušan Matiæ. OFDM as a possible modulation technique for multimedia applications in the range of $\mathrm{mm}$ waves, Introduction to OFDM, II edition 10/30/98/TUD-TVS,pp.1-18

Dusan Matic. OFDM as a possible modulation technique for multimedia applications in the range of $\mathrm{mm}$ waves, 10-30-38/ TUD-TVS, available on-line: www.ubicom.tudelft.nl/MMC/Docs/introOFDM.pdf

Furuzan Atay. $\mathrm{PhD}$ thesis entitled" Cooperative Diversity Relaying Techniques in Wireless Communication Networks", pp.1-199.

Gentian Jakllari, Srikanth, V., Krishnamurthy, Michalis Faloutsos, and Prashant, V., Krishnamurthy. 2007. On Broadcasting with Cooperative Diversity in Multi-hop Wireless Networks", IEEE J. Selected Areas in Communications, Vol. 25, no. 2, pp.1-13.

Gómez-Cuba, F., R. Asorey-Cacheda and F.J. GonzálezCastaño. 2011. A Survey on Cooperative Diversity for Wireless Networks", IEEE Wireless Communications, Vol. X, No. X, pp.1-14.

Gurpreet Kaur and Partha Pratim Bhattacharya. 2011. A Survey on Cooperative Diversity and its Applications in Various Wireless Networks", Int. J. Computer Sci. Engi. Survey (IJCSES), Vol.2, No.4, November 2011, pp.133-152.

Heidi Steendam, Marc Moeneclaey. Optimization of OFDM on Frequency-Selective Time-Selective Fading Channels, available on-line: telin.rug.ac.be/ hs/full/c08.pdf

Howard, H., Xia, Henry, L., Bertoni, Leandro, R., Maciel, Andrew Lindsay-Stewart, and Robert Rowe. 1993. Radio Propagation Characteristics for Line-of -Sight Microcellular and Personal Communications", IEEE Transactions On Antennas And Propagation, Vol. 41, No. 10, pp.1439-1446.
Intzar Ansari, Hemant Soni. 2014. An Extensive Review on Cooperative Wireless Mobile Networks", Int. J. Recent Development in Engineering and Technol, Website: www.ijrdet.com (ISSN 2347-6435(Online) Volume 3, Issue 4, October 2014),pp. 113-116.

Jiang Liu, Wasinee Noonpakdee, Shigeru Shimamoto. 2011. Design and Performance Evaluation of OFDM Based Wireless Services Employing Radio over Optical Wireless Link", Int. J. Wireless \& Mobile Networks (IJWMN), Vol. 3, No. 5, pp.173184.

John S., Seybold. 2005. "INTRODUCTION TO RF PROPAGATION", Published by John Wiley \& Sons, Inc., Hoboken, New Jersey Published simultaneously in Canada, p.3.

Kavita Narayanan, Bilal Shafeek. Performance Analysis of Cooperative OFDM Systems", Int. J. Adv. Res. Electrical, Electronics and Instrumentation Engineering, Vol. 3, Special Issue 3, April pp.162166

Mahmood, A. 2010. Cooperative Diversity in Wireless Networks", J. Engi. Sci. Technol. Rev., 3(1), pp. 184-187.

Mohammed, A., and Z. Yang. 2009. Broadband Communications and Applications from High Altitude Platforms", International Journal of Recent Trends in Engineering, Vol 1, No. 3, pp.239-243.

Monire Norouzi, Alireza Souri, Mohammad esmaeel Akbari. 2012. A new review on Cooperative diversity in wireless networks", Life Sci. J., 2012;9(4):357-361]

(ISSN:1097-8135). http://www.lifesciencesite.com. 55

Munoz, L., and R. Prasad. 2003. WLANs and WPANs towards 4G Wireless, Norwood, MA: Artech House.

Nicholas Laneman, J., David, N.C. 2004. Tse, and Gregory W.," Cooperative Diversity in Wireless Networks: Efficient Protocols and Outage Behavior", IEEE transactions on information theory, vol. 50, no. 12, pp.3062-3080.

Nosratinia, A., T.E. Hunter, A. Hedayat. 2004. "Cooperative Communications in Wireless Networks", IEEE Communications Magazine, pp. 74-80.

Oh-Soon Shin, Albert, M., Chan, H.T. Kung, and Vahid Tarokh. 2007. Design of an OFDM Cooperative Space-Time Diversity System", IEEE transactions on vehicular technol., vol. 56, no. 4, july 2007,pp. 2203- 2215

Pierre Siohan, Cyrille Siclet, and Nicolas Lacaill, Analysis and Design of OFDM/OQAM Systems Based on Filterbank Theory", IEEE Transactions on Signal Processing, Vol. 50, No. 5, pp.1170-1184. 
Prasad, R. 1997. "Wireless Broadband Communication Systems," IEEE Communications Magazine, Vol. 35, p. 16.

Proakis, J.G. 1987. Digital Communications, McGrawHill.

Rajmme Prasad. OFDM for wireless communication system reference book.

Rashmi, R., Sarala, S.M. OFDM: Modulation Technique for Wireless Communication", Int. J. Innovative Res. Adv. Engi.,(IJIRAE) Volume 1 Issue 1 (March 2014) pp. 1-5.

Samuel, C., Yang. OFDMA System Analysis and Design" reference book.

Shahen Shah, A.F.M., Md. Shariful Islam. 2014. A Survey on Cooperative Communication in Wireless Networks", I.J. Intelligent Systems and Applications, 2014, 07, 66-78

Sklar, B. 1997. Rayleigh Fading Channels in Mobile Digital Communication Systems- Part I: Characterization, IEEE Commun. Mag.

Sylvia Ong Ai Ling, Hushairi Zen, Al-Khalid B $\mathrm{Hj}$ Othman, Mahmood Adnan and Olalekan Bello. 2016. A Review On Cooperative Diversity Techniques Bypassing Channel Estimation", Canadian J. Pure and Appl. Sci., Vol. 10, No. 1, pp. 3777-3783, Online ISSN: 1920-3853; Print ISSN: 1715-9997, pp.3777-3783
Tahir, K. 2007. zCoded Cooperative Communications", MSc Thesis, Blekinge Institute of Technology (BTH), Sweden.

Tzi-Dar Chiueh, Pei-Yun Tsai. 2007. book entitled" OFDM Baseband Receiver Design for Wireless Communications", (C) 2007 John Wiley \& Sons (Asia) Pte Ltd. ISBN: 978-0-470-82234-0.

Wenbing Dang, Meixia Tao, Hua $\mathrm{Mu}$ and Jianwei Huang,. Subcarrier-Pair Based Resource Allocation for Cooperative AF Multi-Relay OFDM Systems", work is supported by National Science Key Special Project of China (No. 2008ZX03003-004) and by Huawei Technologies Co., Ltd.

Werner Henkel, Georg Tauböck, Per Ölding. 2002. The cyclic prefix of OFDM/DMT - an analysis, 2002 International Zürich Seminar on Broadband Communications, February 19-21 Zürich, Switzerland.

Zimran Rafique, Boon-Chong Seet and Adnan AlAnbuky. 2013. Performance Analysis of Cooperative Virtual MIMO Systems for Wireless Sensor Networks", Sensors, 13, pp.7033-7052

Zummo, S.A. 2005. "Performance Analysis of Coded Cooperation Diversity in Wireless Networks", Journal of Wireless Communications and Mobile Computing, John Wiley and Sons Ltd.

\section{How to cite this article:}

Anuja Shirke and S.V. Patil. 2017. Analysis, Modelling and Simulation of Co-operative OFDM System to implement Amplify and Forward transmission in wireless Relay Communications. Int.J.Curr.Res.Aca.Rev. 5(1), 8493. doi: http://dx.doi.org/10.20546/ijcrar.2017.501.010 